\section{BMJ Open Respiratory Research}

\title{
Singing for Lung Health: a qualitative assessment of a British Lung Foundation programme for group leaders
}

\author{
Adam Lewis, Phoene Cave, Nicholas S Hopkinson
}

To cite: Lewis A, Cave P, Hopkinson NS. Singing for Lung Health: a qualitative assessment of a British Lung Foundation programme for group leaders. BMJ Open Resp Res 2017;4:e000216. doi:10.1136/

bmjresp-2017-000216

- Additional material is published online only. To view please visit the journal online (http://dx.doi.org/10.1136/ bmjresp-2017-000216).

Received 17 May 2017 Revised 23 June 2017

\section{(a) CrossMark}

NIHR Respiratory Disease, Biomedical Research Unit at the Royal Brompton and Harefield NHS Foundation Trust and Imperial College London, London, UK

Correspondence to Dr Adam Lewis; adam.lewis@imperial.ac.uk

\section{ABSTRACT}

Introduction Singing for Lung Health (SLH) groups are an increasingly popular intervention for people with respiratory disease. There are limited data as to how these groups should be developed and run. We aimed to evaluate the experience of singing leaders both to assess the training provided by the British Lung Foundation (BLF) and to provide information to guide future development of programmes.

Methods A convenience sample of 15 leaders who had received BLF SLH training participated in the BLF service evaluation. Fifteen singing groups were observed, and singing leader interviews and questionnaires were collected. Inductive themes from the qualitative data were the primary outcome. The content of observed singing groups was also rated against the training leaders had received.

Results Singing leaders valued the BLF training but felt that a significant level of expertise is required before joining. Singing leaders often found setting up groups challenging and some found clinician support beneficial. There were important technical aspects of running a lung health group including issues around content, for example, choice of repertoire to suit breathing pattern, and delivery, for example, pace, rhythm and management of group dynamics. Leaders said that group participants reported physical health improvements such as reduced breathlessness on activity. The content and delivery of singing classes observed displayed a good level of fidelity, suggesting that SLH training is effective.

Conclusion The experience of the leaders highlights the requirements, support and technical skills needed to run SLH groups, which have features distinct from generic community singing groups.

\section{INTRODUCTION}

Singing for Lung Health (SLH) is a new but rapidly expanding approach where people with lung disease take part in a group activity that can improve breathing control and posture, without directly focusing on their respiratory limitations. ${ }^{1}$ Participants in SLH groups report that singing helps them to manage their breathlessness, improving quality of life, mood, activities of daily living and participation in meaningful social and

\section{Key messages}

The British Lung Foundation Singing for Lung Health training enables singing leaders to run singing groups for people with respiratory disease competently.

- Singing leaders value the training but can find group set up and delivery challenging

- Important technical aspects of running a Singing for Lung Health group including content and delivery are detailed for the first time. This highlights the distinction between Singing for Lung Health and singing for wellbeing groups.

physical activity. ${ }^{2-5}$ Moreover, SLH group attendance remains high over months up to a year suggesting that is perceived as worthwhile. ${ }^{2}{ }^{3}$ Quantitative data also suggest that SLH improves health-related quality of life. ${ }^{56}$ Chronic obstructive pulmonary disease (COPD) is a leading cause of morbidity and mortality worldwide ${ }^{7}$ with estimated direct National Health Service costs in the UK of $£ 1.8$ billion/year. ${ }^{8}$ Pharmacological treatment of COPD can improve quality of life and lung function, but only to a limited extent, ${ }^{9}$ as can surgical and bronchoscopic treatments. ${ }^{10} 11$ Pulmonary rehabilitation (PR) is a key aspect of high-value ${ }^{12}$ evidence-based care for COPD patients with functional limitation, but access to programmes is variable and adherence and completion is suboptimal. ${ }^{13}$ Furthermore, a defined optimal package for long-term self-management does not yet exist. ${ }^{14}$ In this context, a recent consensus statement and systematic review of SLH has highlighted future research questions, including what aspects of singing training are most important to run an effective group and how singing leaders can best be supported in this role. ${ }^{1}$

Since 2015, The British Lung Foundation (BLF) has provided training and funding to singing leaders to set up and run SLH groups. 
BLF financial support lasts for the initial 12 weeks of group set up. The BLF training programme has developed since 2015, where 1 day of training was provided for singing leaders. Now, leaders receive an initial 2 days of training, a further peer-to-peer training day and a financial stipend for setting up groups and mentoring with an SLH trainer. The BLF training enables leaders to learn the basics of vocal and respiratory anatomy and physiology as well as the pathophysiology of common respiratory diseases. Leaders learn about posture and the use of the whole body in singing and breathing. Leaders learn how to run specific elements of a group, such as physical and vocal warm ups, rhythm and pitch warm ups, breathing exercises, appropriate repertoire and relaxation exercises.

No data exist on the effectiveness of SLH training and how this is reflected in the actual delivery of SLH groups. Furthermore, the experiences of SLH leaders running groups have not previously been researched. By understanding these experiences, technical aspects of running SLH groups may be understood, and differences and similarities can be established in comparison with other generic community choirs. This information is important for establishing the intervention fidelity of SLH during future clinical trials and for future decisions about commissioning SLH as a healthcare intervention.

\section{METHODS}

\section{Service evaluation team}

$\mathrm{AL}$ (male), a physiotherapist, has a $\mathrm{PhD}$ in qualitative research investigating the experiences of patients with COPD who were referred to PR, and then interviewed again after completion, drop out or not starting a programme. Adam led the interviews. PC (female) is a music therapist with prior relationship with the singing leaders. She developed content, resources and led the BLF SLH training, and NH (male) is a respiratory physician who provided academic supervision on the service evaluation. AL was interested in investigating the potential clinical benefits of SLH. PC was interested in how the SLH training programme could be improved for future training programmes. $\mathrm{NH}$ had led two clinical trials of the short-term effects of SLH in patients with COPD, ${ }^{56}$ but had no prior knowledge of content or delivery in relation to the BLF singing programme. SLH leaders were informed that the service evaluation team were investigating their experiences of BLF training and the set up and running SLH groups.

\section{ETHICAL APPROVAL}

This paper includes results from an internal service evaluation for BLF. No institutional ethical committee approval was required for the service evaluation.

\section{RECRUITMENT}

Between June and August 2016, a convenience sample of 19 singing leaders trained by the BLF were contacted by AL and PC via email and 15 of these agreed to participate and provided written consent. The singing leaders had a range of backgrounds. They were choir leaders, adult education teachers, speech and language therapy trained, community musicians, vocal coaches and music teachers. They were interviewed by AL about their experiences undergoing BLF training and running groups. Some groups had stopped running due to funding difficulties or paused over the summer.

\section{DATA COLLECTION}

SLH group leaders completed a questionnaire prior to their interview with AL to provide some background about their experiences (online supplementary file 1).

Fifteen singing leaders were female and one was male. Semistructured interviews took place in the venue where the singing group ran, or at the leader's home, depending on how long the leader was able to use the singing venue. Leaders were asked about their experience of setting up and running a SLH group and their experience of BLF training and resources. Interviews lasted between $26 \mathrm{~min}$ and $44 \mathrm{~min}$. The semistructured interview schedule is included as online supplementary file 2 . No repeat interviews were performed.

Singing groups were observed tojudge the extent to which singing leaders used the techniques from BLF training and the class resources (eg, 'Singing for Breathing' CD). In order for AL to gain sufficient understanding of the BLF SLH training, he first observed all singing leader weekend training sessions that were video-recorded. Six aspects of the singing groups were scored independently by AL and PC:physical warm-up, breathing exercises, vocal warm-ups, rhythm and pitch games, repertoire and relaxation. These were graded from 0 to 10 , with a score of 10 representing best practice. Scores were recorded immediately at the end of the SLH session. Leaders were not informed of the content of the observation scoring system. The six section scores were then added together, and a total of 60 given for each session was observed. A score of 60 meant that the leader displayed practice exactly in accordance with the principles and practice set out in the BLF training. A score of 0 for a component meant that no such component was observed in that group, for example, the absence of a physical warm-up. On each scoring sheet, observation notes were also made by PC and AL. As part of the SLH training, each leader was offered mentoring sessions with PC. Two singing leaders received one of their mentoring sessions on the same day after the group observation, and independent scoring had been completed. After each singing group observation and interview, $\mathrm{PC}$ and $\mathrm{AL}$ then met to compile group scores and have a debriefing session to discuss observations in order to develop recommendations for the BLF to improve its future service delivery of SLH. 


\section{DATA ANALYSIS}

PC had trained the leaders and therefore she did not participate in the interview process. However, as part of each debriefing, $\mathrm{PC}$ put singing leaders' experiences in context with the format of BLF training that each trainer had. Interviews were transcribed verbatim by $\mathrm{AL}$. AL and PC independently coded the data and performed a thematic analysis on the data. ${ }^{15}$ The interview data were put in context with singing group observation field notes (online supplement file 3) and questionnaire data. Codes and themes were discussed with a leader who had received the SLH training but could not be interviewed during the service evaluation. Following this, the results were also shown to a member of a SLH group. Further documentation of the thematic analysis is provided in online supplement file 4. A completed COREQ qualitative research quality criteria checklist is provided in the online supplementary file 5 .

Statistical analysis of group observation scores was performed using SAS V.9.4 statistical analysis package. Due to the low number of group observations and different distributions of each observer's observations, weighted kappa coefficient tests were performed to investigate the level of agreement in scores between AL and PC across the six categories of SLH and the overall scoring. Spearman rank correlation was used to analyse differences in the use of BLF training across groups.

\section{RESULTS}

The themes from the analysis are provided below. Alternate names have been used so that leaders remain anonymous.

\section{Training is valuable but intense}

BLF SLH training is a valuable and intense experience.

\section{I don't think a complete novice will be taken...I think the training is really intense and over a really short amount of time.-Maureen \\ I actually think it was a fantastic 2 days. It was really energising. It was brilliant working intensively with that number of people. The bond you build with people was brilliant.-Eileen}

A certain level of group leading or vocal coaching expertise is recommended before participating in the BLF training programme because of the complexity of running a group.

Following the initial training, onward support is essential.

The mentoring is fantastically useful.-Karen

Being able to ask questions and run through things or

troubleshoot things is definitely very helpful.—Rachael

\section{Group set up}

Groups were set up by singing leaders. This involves finding appropriate venues, marketing the group and recruiting singers. Setting up SLH groups can be challenging. Healthcare professionals, Breathe Easy groups and Clinical Commissioning Groups provided various levels of support across the groups:

Actually quite a lot of our members come from there, probably about 7 or eight come from the breathe easy group.-Toni

However, some leaders perceived these networks as not being proactive or helpful in their support for setting up SLH:

The Breathe Easy groups have been very cold as well and that's the difficulty cos they are right on my doorstep where people live locally who would be $100 \%$ in trying it out.Esme

It may take longer than 12 weeks for a singing group to become properly established and for singing leaders to feel confident in running groups. Interactions with the existing BLF 'Breathe Easy' patient support groups should provide synergy and participants but developing local relationships is important.

I think twelve weeks is a good sort of length of time but once you get to that point it's like, right, let's get going.-Julie

\section{THE BENEFITS OF SLH}

SLH was perceived as a hugely beneficial activity for singers with respiratory disease. Leaders reported that some singers do not want to focus on their lung condition in this setting and simply want to have fun with others in a social setting. The skill of an expert leader enables people to have fun and gain clinical benefit simultaneously.

The thing I really like in this room that I haven't seen in any of the other classes, is that we don't keep talking about our breathing problems, 'I don't want to, I want to just do, I want to improve my breathing through doing'.-Maureen

SLH leaders create a new culture where individuals feel connected with one another socially within the shared task of singing, experiencing the rhythm and tempo of the breath and body in music.

You are creating a new culture for people.-Julie I always do the relaxation thing so it feels a bit meditative in a way... it brings people into their bodies and into the room. I could do that in community choirs but often it doesn't feel like it's really the right thing to do but here it really does, it feels really right to bring the group together each week, be in the space, think about the body...-Colin

This 'new culture' is one of social bonding in a task with a shared purpose and creative product. These experiences will be novel for many with respiratory disease.

By providing an atmosphere of safety and comfort, people are allowed to focus on themselves and others within a group, as respite from their condition. 
Having a bit of a respite where you enjoy something and you're present in the moment and you are not worrying about what's coming next has a real positive impact.—Eileen

Through increased self-awareness, people can learn to cope with the loss of a previous 'well' self.

That relationship between who you feel you are and your physical body can kind of come dissociated when you are poorly.-Rachael

When that self-awareness comes, it's really valuable. You know it's the key to change.-Claire

I think there is something powerful in it which goes beyond the practical, physical, it's you know to do with the personcentred, themself and the life within them.-Kirsten

Singing leaders have also reported many health improvements in participants who have sung in their groups.

The nurse had...put it down to the singing that was something that was improving their lung function.-Colin It's that kind of mental well-being aspect and then actually from singing you get that great rush of endorphins and it makes you more alert.-Toni

\section{SLH is complicated}

Singing leaders provided great detail about the complicated nature of SLH. There is a skill to adapting repertoire for degrees of breathlessness, and the session should be performed with musicality and rhythm throughout.

Them thinking they won't be able to get to that third phrase, whereas one phrased of the same length they are doing (it)... so just kind of playing about with the repertoire a bit.Eileen

It's actually encouraging people to use their bodies and their breath in a way that's helpful for them, without them paying too much attention to that as the outcome.-Carly

Providing a 'safety net' of a simple repertoire where the group do not feel too challenged initially allows individuals to flourish within a group. The skilled leader can then develop this repertoire by adding rounds or harmonies for example.

\section{No one should ever fail.--Janice}

Songs sung with simple repertoire, in a call and response format, were often seen as effective. If singers use handouts for complicated lyrics, attention should be directed towards any changes in posture as a result and advice to correct poor posture is advised:

\footnotetext{
African songs, they are very accessible...People haven't generally done them before and um they are usually very rhythmic, so very diatonic so they are using very very simple tune, almost like nursery rhymes you know, they are very primary harmony easy to do, really easy to pick up and very rhythmic usually...they do often incorporate movement as well...-Rachael
}

My experience is you do all of this movement and getting people moving and breathing and you give people a song sheet and they hold onto it and they are looking down at it and they have closed their windpipe and they're not singing very well.-Julie

Singing in call and response because you don't have to worry about the words and you don't even have to remember what's happening next, you just hear and sing back and that, that is actually quite liberating.-Kirsten

The pace of running SLH groups often needs to be slower than other community singing groups. When leaders increase the difficulty of repertoire or add harmonies, people may struggle. Volunteers are seen as helpful, helping with room set up, administration or leading half a group in a round.

I did another song which was too hard in terms of harmony. Lovely song, I would say perfect long sort of pushy notes in $i t$, but it wasn't a good choice.—Laura

You need some other singing volunteers to support and still go at the same pace as the people in the group who are there... to support them you know when you are doing all the exercises and things if you are in a group of eight people doing it you kind of don't feel so vulnerable.-Julie

\section{SLH'S ROLE AS A TREATMENT}

Singing leaders felt that a patient referral system may reduce the burden of group set up and running a group. Those who received clinical support within groups appreciated this. However, it is not clear whether SLH groups should be funded on the NHS because the intervention offered may be altered as a result. Social prescribing, charitable grants and patient donations were funding routes used following the 12-week BLF funding period.

When things have been taken in house (NHS) they often then come under a number of constraints that aren't running it in a voluntary and community sector setting or a private sector setting, so it would probably end up being a 10week programme that then people got kicked off.-Eileen

If there's something actually wrong with somebody at that moment (the respiratory nurse) will actually take it and he is actually creating that link now essentially between what's happening in the community and what's happening in the hospital.-Janice

The second quote is an example where a clinician helped when a group member was experiencing an exacerbation. Having healthcare professionals (HCP) attend the group can help singing leaders working independently and at times are not prepared for or feel appropriate within their remit to talk about medical conditions or deal with exacerbations.

\section{OBSERVATION SCORES}

Overall, the BLF training has prepared the leaders to run singing groups to an acceptable standard. However, there was significant variation of practice between groups: the 


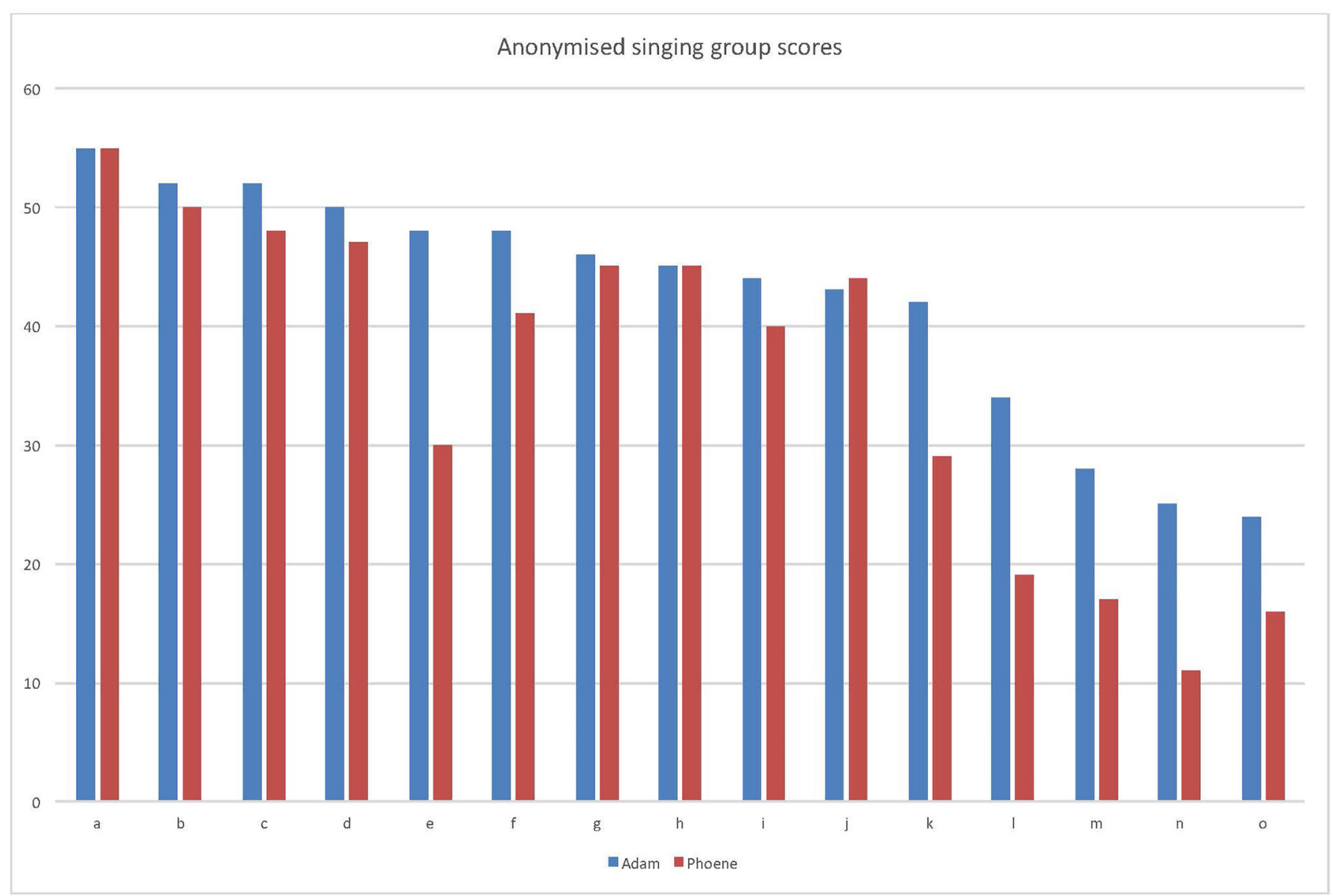

Figure 1 SLH group observation scores: the graph shows each group score marked out of 60 based on PC's and AL's independent observations. Nine out of the 15 groups achieved a score of 40 or more by both observers.

ranking of the groups was very well matched (R: 0.91) between observers as shown in figure 1.

The scores above show good overall consistency of the training that leaders received transferring into their practice during the observations. The bar chart above suggests $\mathrm{AL}$ gave higher scores than $\mathrm{PC}$ across groups. Further analysis was needed to determine the significance of the differences in independent scoring between observers. Weighted kappa coefficient tests were performed on each of the six group categories (ie, repertoire) comparing scores from each observer. Overall, there is moderate agreement $(\mathrm{K}=0.42)$ between $\mathrm{PC}$ and $\mathrm{AL}$ in individual scores across the six categories shown in figure 2 below. Indeed, PC's lower and more nuanced observations might reflect her music therapy expertise, and greater experience of SLH, because of her involvement in the development of the SLH training.

The highest level of agreement was in the rhythm and pitch warm ups $(\mathrm{K}=0.72)$, and the lowest level of agreement was in the physical warm ups $(\mathrm{K}=0.21)$. Consequently, a workshop on physical warm-ups was included in a 'Train the trainer' weekend for future rounds of training, and a new standard of practice was agreed between all new trainers.

\section{DISCUSSION}

Main findings

The singing training provided by the BLF allows leaders to feel competent and confident in running SLH groups, although a previous level of expertise is recommended because of the complicated nature of running such groups. Leaders felt that they were able to provide a safe environment for singers to have respite from living with their condition, while simultaneously reporting that they observed health benefits in participants.

Our observational data also show that leaders' delivery of SLH in practice is consistent with the training that they have received. Differences in observer scores have been used to provide recommendations for the BLF to improve their training programme further.

\section{Significance of findings}

Setting up singing groups is perceived to be a challenge. The BLF provide a financial stipend for group set up and further payment per session led. However, leaders may require further financial support. Groups being supported by singers' donations may be a sustainable option. However, this may exclude people from lower socioeconomic backgrounds, leading to inequality in 


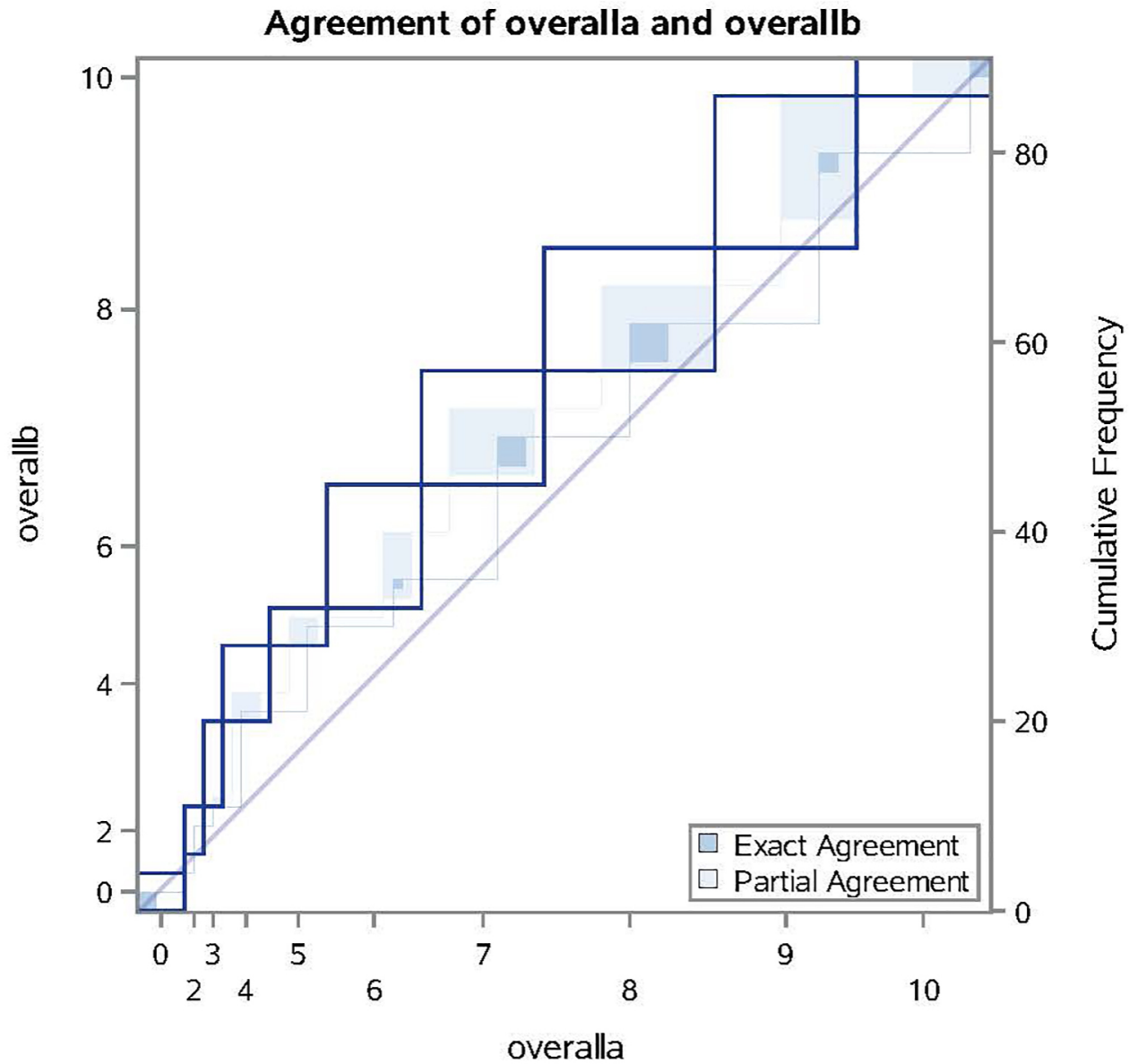

Figure 2 Overall weighted kappa coefficient agreement: this graph illustrates the overall agreement between observer A $(P C)$ and $B(A L)$.

availability for those less well off. Leaders reported pros and cons for SLH groups being embedded within an NHS care pathway for respiratory patients. A referral system may help recruitment and ease the burden of set up. Clinical support is also beneficial to allow singers to focus on running each session effectively and deal with singers who are having an acute exacerbation. However, singers do not necessarily want to be treated as 'patients' in this setting and may well not attend groups if exacerbating. Furthermore, singing groups may not operate on a rolling basis with limited funding in the NHS. This could be problematic for patients who feel part of a new culture and engaged in a meaningful community activity.

SLH is complicated and could be viewed as a multicomponent self-management intervention. Leaders require sensitive judgement to adapt repertoire for individuals' or groups' levels of breathlessness. Repertoire that works is simple. The use of chant-like formats that stimulate additional physical activity (such as clapping or dancing/ swaying) and can easily be taught in a call-and-response format and easily adapted into rounds is recommended.

Anyone in the UK can call themselves a singing teacher, vocal coach or community choir leader. No training is mandated, and there can be a diverse understanding of pedagogy, vocal anatomy as well as physiology and vocal leadership skills. Some trained vocal coaches may not have the skillset to run group singing teaching repertoire 'by ear'. Some community choir leaders may be intuitively musically adept, but know nothing about how the voice works, and many singing teachers have incorrect assumptions about how we breathe to sing. Some will not know how to plan sessions, others may be inflexible to responding spontaneously when needed. Many singing leaders will have had no information about lung disease from respiratory specialists prior to running the groups. Further discussion on this is provided by Cave. ${ }^{16}$ The singing leader competencies required to run SLH groups are included in figure 3.

SLH enables people to feel part of a new culture where they are not treated as patients but may still benefit clinically. Being in the right space and being cared for has previously been reported. ${ }^{2}$ These data provide further information that people with respiratory disease benefit from having a space where their condition is not talked about as a disease, and their breathlessness is treated through song. Previous studies have also suggested physical, social and emotional benefits from joining an SLH group. ${ }^{3-5}$ However, these potential improvements in breathlessness, or the control of breathlessness during a flare up, or a 


\begin{tabular}{|l|l|}
\hline 1 & An understanding of vocal anatomy and physiology (as well as basic whole body anatomy). \\
\hline 2 & An understanding of the art and science of singing teaching. \\
\hline 3 & Lesson planning and reflective practice. \\
\hline 4 & Structure and spontaneity. \\
\hline 5 & A broad repertoire of appropriate songs. \\
\hline 6 & How to run physical, breathing, vocal and rhythm and pitch warm ups. \\
\hline 7 & An understanding and ability to demonstrate how we breathe to sing. \\
\hline 8 & A holistic understanding of the kinaesthetics and whole body proprioception in breathing. \\
\hline 9 & An understanding and ability to talk about the psychology of breathing. \\
\hline 10 & Knowledge of lung health and lung disease. \\
\hline
\end{tabular}

Figure 3 Singing for Lung Health leader competencies: these competencies are required of any new singing leader in order to run a Singing for Lung Health Group effectively.

reduction in chest infections have yet to be investigated in large randomised controlled trials.

Our findings highlight that running an SLH group is different from a more generic singing for well-being or general community choir. Specialist training is required to be competent because of the complexities of SLH group set up, fostering personal development in a group of individuals with respiratory disease and technical aspects of running a group effectively. Our data provide technical details on how to run such groups effectively and the importance of creating a specialist approach to delivery. It is not clear whether SLH groups would be best situated within the National Health Service. If SLH were to be adopted more broadly within the UK in the current healthcare climate, more research is required that suggests significant clinical benefits in larger patient cohorts.

SLH is a group intervention. As such it will likely be compared with PR. However, there are significant differences between SLH and PR. Compared with PR, less structured support is available for individuals running SLH groups, and leaders often run groups independently, as freelancers alongside other paid work. Support networks for leaders and singers need to be developed through a needs-led approach, in COPD 'hotspots' for example, with locality champions providing expertise and connections between commissioners, healthcare professionals, NHS Trusts and patient-led groups. SLH leaders feel that they would not be able to run such groups competently without the training provided by the BLF. Compared with PR, which only recently completed a nationwide audit of services, ${ }^{17}$ this service evaluation of SLH is occurring during the foundation years of a developing intervention.

Since this service evaluation began in April 2016, there has been an expansion of SLH groups around the country. Now there are 35 BLF SLH groups in the UK out of a total of 80 groups known to the BLF as shown in figure 4. Further networking between BLF SLH and Breathe Easy groups may increase patient participation in both groups.

\section{Methodological considerations}

The data presented are from a sample of just over half of the BLF-affiliated singing leaders who were still running 


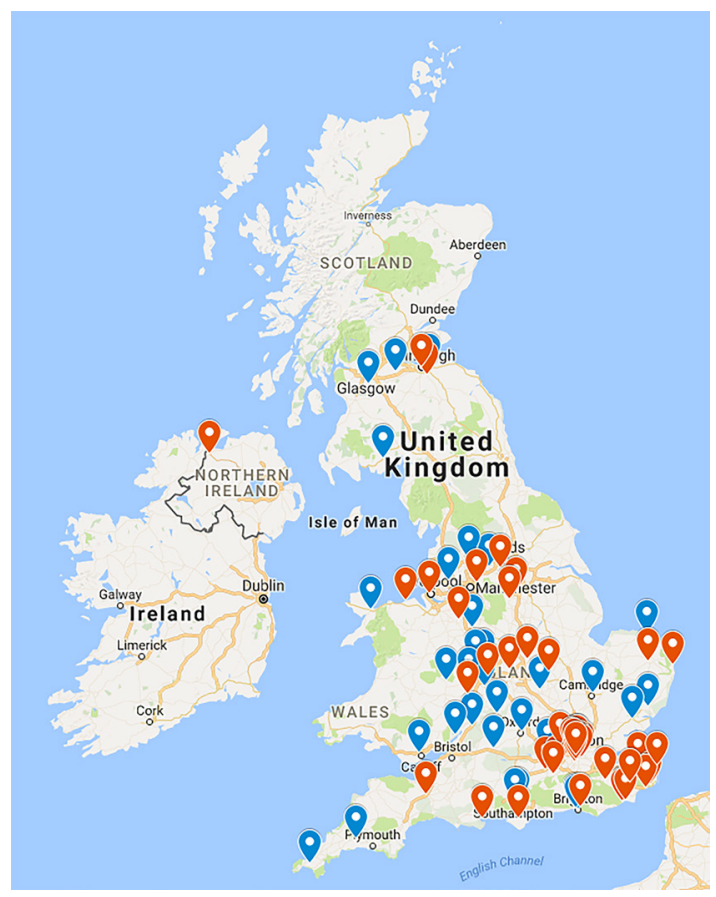

Figure 4 UK Singing for Lung Health groups: the blue points on the map represent groups run by a BLF trained leader. Red points on the map are groups run by nonBLF-affiliated groups. Eleven of these groups are affiliated with Breathe Easy groups. Courtesy of the British Lung Foundation (map data @2017 GeoBasis-DE/BKG (@2009), Google).

groups at the time of the evaluation. The experience of other BLF leaders in the other groups and groups led by those without any specific training may be different. However, it was agreed by AL and PC that data saturation was reached due to no new themes emerging from the final interviews. This was supported by a review of the data by a singing leader who received the BLF training but was not interviewed and a singing group member. Transcripts were not returned to participants.

SLH leaders may have felt obliged to report positive responses because their funding and support was provided by the BLF. All participants were encouraged to be open and report both positive and negative experiences. The data presented suggest that the interview process allowed for this. PC was part of the evaluation team. As such she was an 'insider' within the evaluation. ${ }^{18}$ She was involved with mentoring leaders at the time of the service evaluation. Therefore, she was not present during the interviews but was an important participant in the evaluation team having designed the training programme. She was therefore able to assess how the training provided for each participant reflected the practice that was observed during group visits. Before this evaluation, PC had not visited leaders to observe their practice. Her intention was to improve the training programme. AL was an 'outsider' in SLH to balance the evaluation team. He had observed and joined in an SLH group once prior to the evaluation. The use of a multidisciplinary team when investigating non-clinical interventions for medical conditions is desirable. ${ }^{19}$

\section{CONCLUSION}

SLH is a developing method of running singing groups for individuals living with respiratory disease, with increasing evidence that patients value the experience. Singing leader accounts of the training provided by the BLF, and the further set up and delivery of SLH highlight the requirements, support and technicalities needed to run a group successfully. The specialised approach to SLH training and delivery distinguishes these groups from generic community singing groups. These data should provide reassurance that it is possible to develop and maintain a best practice standard. This is important for future delivery and expansion of SLH across service providers.

Further large-scale randomised controlled trials are needed prior to SLH groups becoming adopted as an evidence-based self-management option for commissioners to fund more widely.

Acknowledgements Thanks to Jay Dowle, who has provided editorial input from his perspective as a participant in an SLH group, and Emily Foulkes, an SLH leader who also provided comments on the paper. Thanks to Dr Emil Walsted for statistical consultation.

Contributors AL, PC and NSH designed the service evaluation, semistructured interview schedules and questionnaires. AL performed the semistructured interviews. AL and PC analysed the data. AL drafted the initial manuscript, and all authors worked on the final revision. AL agrees to be accountable for all aspects of the work in ensuring that questions related to the accuracy or integrity of any part of the work are appropriately investigated and resolved.

Funding This work has been funded by a $£ 25000$ grant from the British Lung Foundation

Competing interests AL reports, other than the British Lung Foundation (BLF) service evaluation funding, that he has received consultation fees for providing content and delivery of Singing for Lung Health Training since the completion of the service evaluation. PC reports, other than the BLF service evaluation funding, that she has created and delivered all Singing for Lung Health training including the training delivered to participants in this service evaluation. NH declares no competing interests.

Provenance and peer review Not commissioned; externally peer reviewed.

Data sharing statement Other British Lung Foundation Singing for Lung Health service evaluation data have been written in an internal report to the British Lung Foundation.

Open Access This is an Open Access article distributed in accordance with the Creative Commons Attribution Non Commercial (CC BY-NC 4.0) license, which permits others to distribute, remix, adapt, build upon this work non-commercially, and license their derivative works on different terms, provided the original work is properly cited and the use is non-commercial. See: http://creativecommons.org/ licenses/by-nc/4.0/

C Article author(s) (or their employer(s) unless otherwise stated in the text of the article) 2017. All rights reserved. No commercial use is permitted unless otherwise expressly granted.

\section{REFERENCES}

1. Lewis $A$, Cave P, Stern M, et al. Singing for lung Health-a systematic review of the literature and consensus statement. NPJ Prim Care Respir Med 2016;26:16080.

2. McNaughton A, Aldington S, Williams G, et al. Sing your lungs out: a qualitative study of a community singing group for people with chronic obstructive pulmonary disease (COPD). BMJ Open 2016;6:e012521.

3. Morrison I, Clift S, Page S, et al. A UK feasibility study on the value of singing for people with chronic obstructive pulmonary disease 
(COPD). UNESCO Observatory Multi-Disciplinary Journal in the Arts. 2013;3:1-19.

4. Skingley A, Page S, Clift S, et al. 'Singing for Breathing': Participants' perceptions of a group singing programme for people with COPD. Arts Health 2014;6:59-74.

5. Lord VM, Hume VJ, Kelly JL, et al. Singing classes for chronic obstructive pulmonary disease: a randomized controlled trial. BMC Pulm Med 2012;12:1-7.

6. Lord VM, Cave P, Hume VJ, et al. Singing teaching as a therapy for chronic respiratory disease-a randomised controlled trial and qualitative evaluation. BMC Pulm Med 2010;10:41.

7. Lozano R, Naghavi M, Foreman K, et al. Global and regional mortality from 235 causes of death for 20 age groups in 1990 and 2010: a systematic analysis for the global burden of disease study 2010. Lancet 2012;380:2095-128.

8. British Lung Foundation. Estimating the economic burden of respiratory illness in the UK, 2017.

9. National Institute for Clinical Excellence. Management of chronic obstructive pulmonary disease in adults in primary and secondary care (partial update). This guideline partially updates and replaces NICE clinical guideline 2010;12. http://guidanceniceorguk/CG101

10. Zoumot Z, Davey C, Jordan S, et al. Endobronchial valves for patients with heterogeneous emphysema and without interlobar collateral ventilation: open label treatment following the BeLieVeRHIFi study. Thorax 2017;72.
11. Davey C, Zoumot Z, Jordan S, et al. Bronchoscopic lung volume reduction with endobronchial valves for patients with heterogeneous emphysema and intact interlobar fissures (the BeLieVeR-HIFi study): a randomised controlled trial. Lancet 2015;386:1066-73.

12. Zoumot Z, Jordan S, Hopkinson NS. Emphysema: time to say farewell to therapeutic nihilism. Thorax 2014;69:973-5

13. Rochester CL. An Official American Thoracic Society/European Respiratory Society Policy Statement: enhancing implementation, use and Delivery of Pulmonary Rehabilitation. 2015;192:1373-86.

14. Jolly K, Majothi S, Sitch AJ, et al. Self-management of health care behaviors for COPD: a systematic review and meta-analysis. Int $J$ Chron Obstruct Pulmon Dis 2016;11:305-26.

15. Braun V, Clarke V. Using thematic analysis in psychology. Qual Res Psychol 2006;3:77-101.

16. Cave P. Singing with one voice?: London Arts in Health Forum Blog, 2017. https://lahf.wordpress.com/2017/03/22/singing-with-onevoice/ (accessed date 16 June 2017).

17. Steiner M, Holzhauer-Barrie J, Lowe D, et al. Pulmonary Rehabilitation: Steps to breathe better National Chronic Obstructive Pulmonary Disease (COPD). Audit programme: clinical audit of pulmonary rehabilitation services in England and Wales 2015, 2016.

18. Pringle J, Hendry C, McLafferty E. Phenomenological approaches: challenges and choices. Nurse Res 2011;18:7-18.

19. Gick ML, Nicol JJ. Singing for respiratory health: theory, evidence and challenges. Health Promot Int 2016;31. 